\title{
Análisis y evaluación de la antena dipolo meandro
}

\author{
Juan G. Estrada*, Carlos I. Páez-Rueda ${ }^{* \S}$ \\ *Grupo SISCOM, Departamento de Electrónica, Pontificia Universidad Javeriana, Bogotá. \\ §email: paez.carlos@javeriana.edu.co
}

(Recibido: Marzo 3 de 2010 - Aceptado: Junio 1 de 2010)

\begin{abstract}
Resumen
En este artículo se presentan las curvas de diseño y la evaluación de la antena meandro construido con alambres en cuatro diferentes topologías con el objeto de ser diseñada para aplicaciones que requieran miniaturización. Se utilizó el kernel electromagnético NEC, a través del cual se construyeron diversas curvas de diseño que permiten encontrar los valores apropiados para el correcto funcionamiento de la antena. Las curvas de diseño fueron validadas por medio de la construcción y caracterización de cuatro diferentes prototipos, diseñados para un porcentaje de miniaturización del $50 \%$ comparado con un dipolo resonante lambda medios $(\lambda / 2)$. La impedancia de entrada, el patrón de radiación y la ganancia máxima de la antena fueron evaluados experimentalmente en un punto de operación común a una frecuencia fija de VHF.
\end{abstract}

Palabras Claves: Antena dipolo, Antena meandro, Código electromagnético numérico

ELECTRONIC ENGINEERING

\section{Analysis and evaluation of a meander dipole antenna}

\begin{abstract}
The design curves and evaluation of a wire meander antenna in four different topologies with the aim to be designed for miniaturization applications is presented. A variety of parametric curves were raised with NEC (Numerical Electromagnetic Code), in order to find the appropriate values for the antenna operation. The design curves were validated through the construction and characterization of four different prototypes, designed for miniaturization of $50 \%$ compared with a dipole $(\lambda / 2)$. The input impedance, radiation pattern and maximum gain of the antenna were experimentally evaluated in a common operating point to a fixed frequency of VHF.
\end{abstract}

Keywords: Dipole antenna, Meander antenna, Numerical electromagnetic Code 


\section{Introducción}

Como parte fundamental e inherente de cualquier tecnología inalámbrica, el transductor electromagnético diseñado para transmitir o recibir las ondas electromagnéticas en una determinada dirección cumple un papel fundamental en la adaptación de la energía de una forma guiada a una forma no guiada. Este transductor, llamado antena, tiene una diversidad de desafíos en su diseño que van desde la eficiencia electromagnética con que se transforma la onda hasta los diversos aspectos de conveniencia en la forma, el tamaño y el peso en el contexto de las diversas aplicaciones de las telecomunicaciones donde son necesarios.

En particular, el desafío de disminuir el tamaño de una antena conformada o construida a partir de alambres es de gran relevancia actual, debido a que muchas de las aplicaciones en las bandas de HF, VHF y UHF, requieren una facilidad de portabilidad y mimetización. Como ejemplos de este tipo de tecnologías se encuentran la televisión, el radio, los sistemas de banda ciudadana y algunos sistemas troncalizados como los utilizados por los servicios de radio taxi. Sin embargo, la reducción del tamaño de una antena tiene consecuencias inevitables en el deterioro de la eficiencia de radiación, el cambio de su patrón de radiación y la disminución de su ancho de banda, entre otros.

Diversos métodos para la reducción del tamaño de una antena han sido explorados en la literatura. Entre los más relevantes se encuentran colocar cargas reactivas y resistivas en diversos puntos de la estructura, ya sean concentradas como en Boag et al. (1996) o sean distribuidas como en Altshuler \& Linden (1997) y Simpson (2004), recubrir el alambre con un dieléctrico particular o especial como en Yamada \& Michishita (2005) y en Erentok \& Ziolkowski (2008) y aprovechar el espacio de la estructura al cambiar la forma de las antenas como en Altshuler (2002) y Hosung Choo et al. (2005).

En esta última técnica, se buscan geometrías que a través del llenado del espacio bidimensional (Best 2003) o espacio tridimensional (Hosung Choo et al. 2005, Petko \& Werner 2004), se logre compactar la antena al reducir el largo de la misma. Con esta técnica, el problema es particularmente complejo ya que cambiar la forma de la antena implica cambiar todas las propiedades de radiación de la estructura, y por ende, sus medidas de desempeño. Con la técnica de llenado del espacio, las geometrías fractales han sido exploradas intensivamente en la última década (Werner \& Ganguly, 2003) en especial con tecnologías planares debido a su facilidad de construcción. Ejemplos relevantes de este tipo de antenas se encuentran en la aplicación de la curva Koch en Baliarda (2000) y la curva Peano en Jinhui Zhu et al. (2004). Aún lo anterior, como se concluye en Gonzalez-Arbesu et al. (2003), algunas geometrías de llenado del espacio basado en geometrías tradicionales euclidianas, menos sofisticadas que sus equivalentes fractales, han aprovechado el llenado del espacio para reducir también la longitud de la antena con formas geométricas más simples, que en algunos casos han superado la eficiencia de radiación de sus equivalentes fractales.

En particular, la forma geométrica euclidiana alternativa basada en un meandro fue presentada originalmente por Rashed \& Tai (1991), como alternativa para la reducción de tamaño de una antena impresa monopolo. El nombre de la antena fue planteado por los autores, dado la similitud de la forma básica propuesta con un meandro o curvas que describen el curso de un rio. Este tipo de antena ha sido utilizada en la literatura moderna en diversas configuraciones planas especialmente en antenas impresas monopolo como en Wen-Yan Yin et al. (2009), algunas antenas impresas dipolo como en Rashed-Mohassel et al. (2009) y recientemente analizadas para las aplicaciones de RFID como en Galehdar et al. (2009). Aunque menos frecuente, también ha sido explorada esta geometría en antenas conformada por alambres con el objetivo de lograr miniaturización para las bandas de $\mathrm{HF} / \mathrm{VHF}$, como la presentada en Warnagiris \& Minardo (1998).

En el presente artículo se encuentran las curvas paramétricas de diseño de cuatro antenas dipolo 
en la configuración meandro detallada en la Figura 1 , modelados por medio de alambres conductores y su evaluación a través de cuatro prototipos diferentes. Se escogió la tecnología de antenas basada en alambre, con el objeto de analizar la factibilidad industrial de utilizar este tipo de antenas miniaturizadas en las aplicaciones de la banda de HF/VHF. A continuación, se presentará la metodología utilizada en el presente proyecto de investigación. Posteriormente se mostrarán los resultados de diseño de este tipo de antenas a través de la presentación de diversas curvas de diseño. Adicionalmente se presentarán los resultados experimentales de la evaluación de cuatro prototipos diseñados y construidos con este tipo de forma a una frecuencia fija en VHF. Finalmente, se presentarán las conclusiones y prospectiva del trabajo realizado.

\section{Metodología}

El presente proyecto de investigación se dividió en tres fases metodológicas. La primera fue la generación de las curvas paramétricas que permiten diseñar las antenas tipo meandro. Para esta fase se utilizó el código electromagnético NEC-2 (Numerical
Electromagnetic Code versión 2) distribución pública para el sistema operativo DOS como base computacional electromagnética (Burke \& Poggio 1981). Para utilizar estas rutinas, se debieron desarrollar algoritmos con propósitos académicos en MATLAB, que permitieran generar el modelo de la antena, ejecutar por línea de comandos las rutinas de NEC-2 y extraer posteriormente los datos de interés. Las curvas de diseño de las diversas configuraciones de la antena dipolo meandro fueron realizadas a través del cálculo electromagnético de la antena considerando las variaciones explícitas de los parámetros L y W definidas en la Figura 1. Las curvas de diseño consisten de gráficas bidimensiones donde se detallan a través de iso contornos la directividad máxima, la parte real y la parte imaginaria de impedancia de entrada de la antena. Adicionalmente se consideraron las modificaciones de $\Delta \in[0.015 \lambda, 0.02 \lambda] \mathrm{sin}$ obtener resultados diferenciadores en las curvas de diseño. Variaciones más pequeñas de $\Delta$ no fueron consideradas, debido a las posibles dificultades de fabricación de los
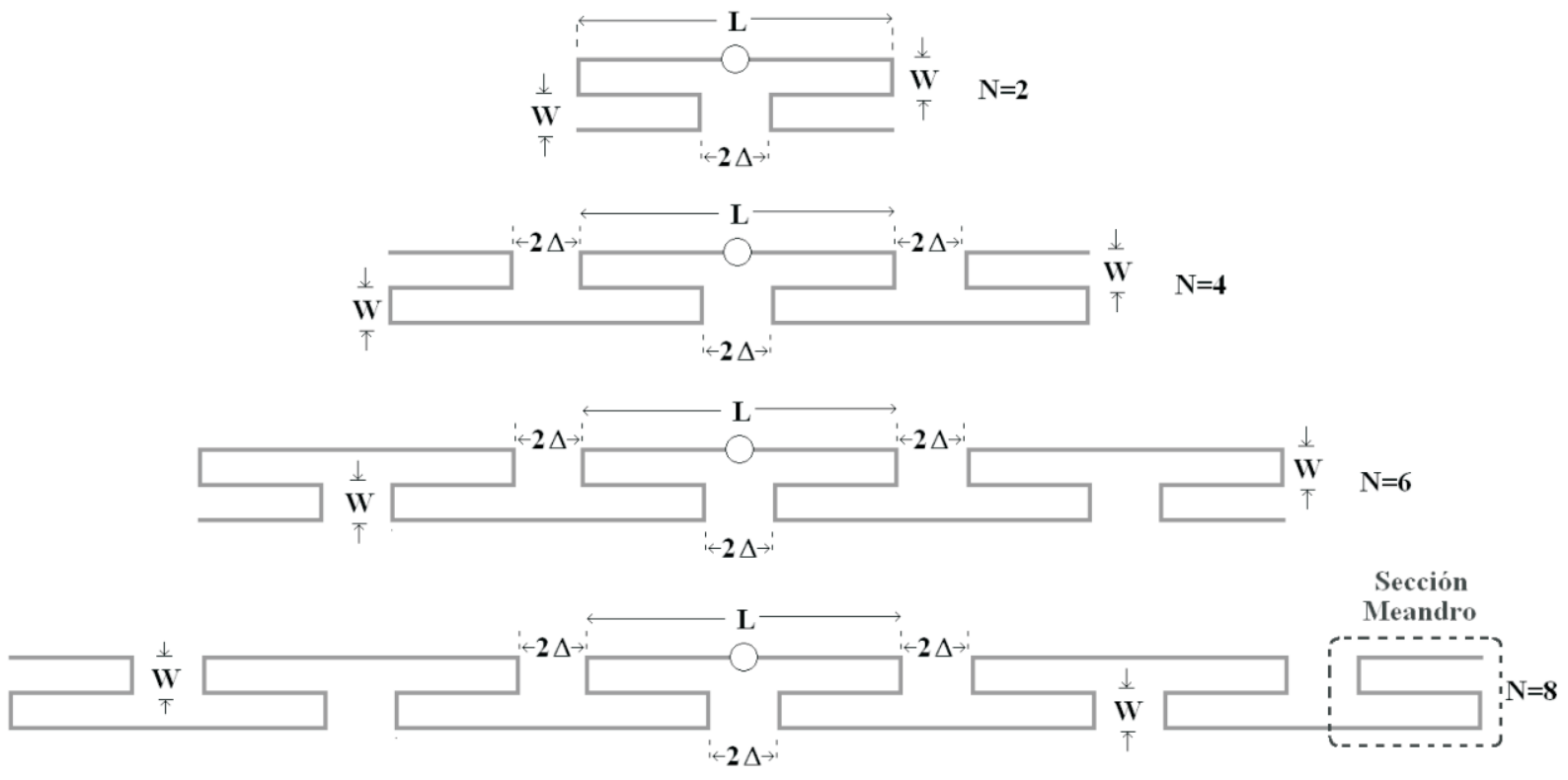

Figura 1.Forma geométrica de un dipolo en configuración meandro 
prototipos y debido a que la hipótesis de una corriente axial uniforme no es correcta para alambres demasiados cercanos, y por ende, el código electromagnético NEC-2 no es apropiado como base computacional. El radio del alambre considerado en todas las curvas fue fijo a un valor de $a=0.00119 \lambda$.

En la segunda fase de este proyecto de investigación se procedió a diseñar y construir cuatro diferentes prototipos de las antenas meandro, bajo la consideración de comprobar la capacidad de compactación de la antena y la factibilidad de su fabricación. En el diseño se definieron los puntos de operación apropiados para poder comparar efectivamente los diversos prototipos construidos a una frecuencia de $150 \mathrm{MHz}$. La escogencia de la frecuencia de operación fue debido a la facilidad tener un Balun de baja pérdida y a la facilidad de construir un dipolo resonante $\lambda / 2$ para efectos de comparación. Los puntos de operación en las curvas paramétricas construidas fueron aquellos que permitiera disminuir la longitud total de la antena meandro al cincuenta por ciento $(50 \%)$ comparado con el dipolo ideal resonante $\lambda / 2$.

También se consideró para el diseño, que los dipolos meandros estuvieran operando en su resonancia serie, con el objeto de poder comprobar este comportamiento resonante de la antena. Este punto de operación es relevante, dado que facilita industrialmente la construcción de redes acople para las antenas. Para la fabricación de las antenas se definió un conjunto de procedimientos, formas y ensambles mecánicos que permitieran tanto la unión al mástil como soporte mecánico resistente a las condiciones ambientales presentes en el set de medición. El proceso de fabricación fue manual, con el objeto de disminuir los costos involucrados en el proceso, y a su vez, evaluar las fallas esperadas bajos estas condiciones de fabricación.
En la tercera fase de este proyecto de investigación, se procedió a definir y realizar las medidas experimentales de los parámetros de las antenas meandro construidas. Estas medidas de emérito fueron la impedancia de entrada, la ganancia máxima y el patrón de radiación en el plano E y $\mathrm{H}$. Para la medición de la impedancia de entrada de la antena se coloco la antena bajo prueba (DUT) en un mástil neumático/hidráulico de 10 metros ilustrado en la Figura 2.a y a través de un cable coaxial previamente caracterizado se midió la impedancia de entrada de la antena con un analizador vectorial de redes referencia 8714ES. Esta impedancia experimental presentada en este documento, corresponde al sistema Balun/Antena, debido a la imposibilidad tecnológica de medir los parámetros de desempeño del Balun con ese equipo. Para medir el patrón de radiación y la ganancia máxima en el plano de $\mathrm{E}$ y $\mathrm{H}$, se colocó el DUT en un mástil y un dipolo resonante previamente caracterizado en otro mástil, con la apropiada polarización separada por una distancia de 7 metros, como se ilustra en las Figura 2.b y Figura 2.c. Posteriormente se realizó un barrido cada diez grados del DUT y se procedió a adquirir los datos correspondientes. Por medio del procedimiento de dos antenas documentado en Balanis (1997) y con las características previas de los cables y conectores, se procedió a determinar esas medidas de desempeño.

\section{Resultados y discusión}

\subsection{Curvas paramétricas del dipolo meandro $\operatorname{con} \mathbf{N}=2$}

En la Figura 3 se ilustra la curva paramétrica de la impedancia de entrada de la antena meandro $\mathrm{N}=2$. En esta gráfica, en la primera banda de grises de izquierda a derecha se encuentra la primera resonancia serie, la cual se ha detallado por medio de una línea continua negra en dicha figura. El cambio abrupto entre el color blanco y negro representa la primera resonancia paralelo y la 


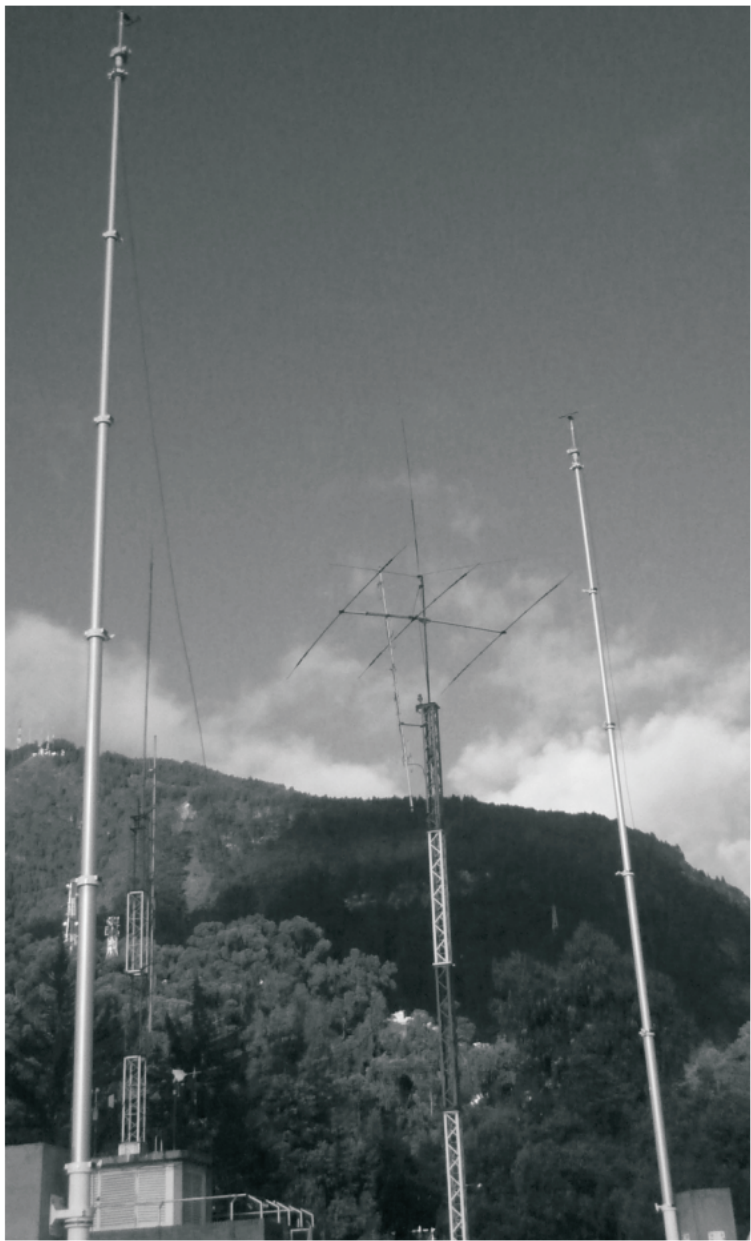

(a)

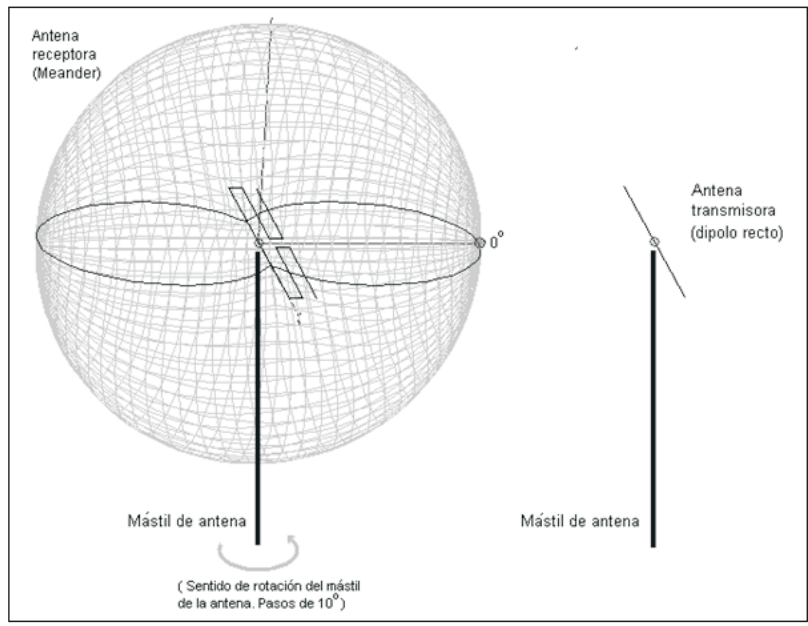

(b)

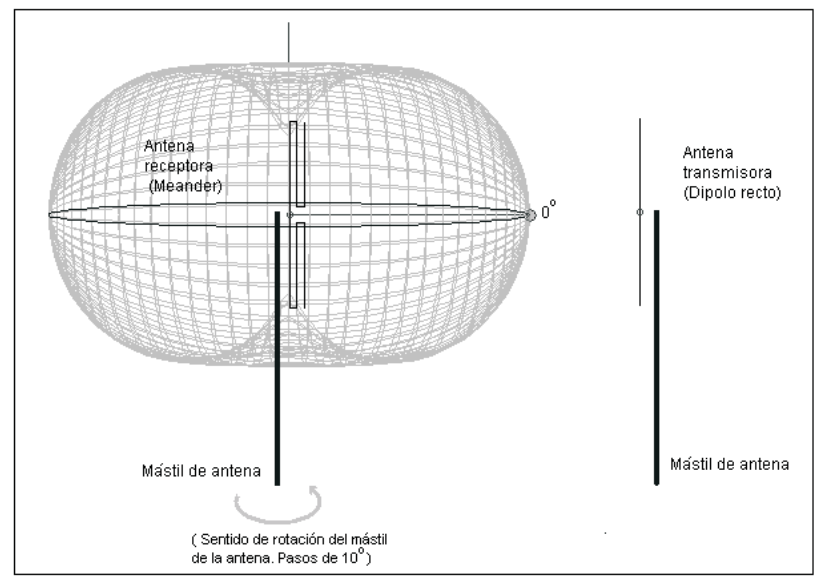

(c)

Figura 2. Configuración experimental para la mediación de las medidas de desempeño de las antenas meandro construidas. (a) Mástiles neumáticos/hidráulicos. (b) Configuración de medición del patrón E. (c) Configuración de medición del patrón H.

segunda banda de grises corresponde a la segunda resonancia serie de esta antena. Esta gráfica muestra que la primera resonancia serie se encuentra entre $0.17 \lambda \leq L \leq 0.26 \lambda$ mostrando una capacidad de reducción de tamaño con respecto a un dipolo resonante $\lambda / 2$ entre el $34 \%$ y el $52 \%$. Esa misma gráfica demuestra que reducir el largo de la antena con la topología meandro tiene como consecuencia una reducción en su impedancia de resonancia, la cual pasa de un valor típico de $50 \Omega$ para un dipolo recto resonante a $Z_{\text {in }} \in[7 \Omega, 18 \Omega]$. Un análisis gráfico de la relación entre W y L en la primera resonancia serie de esta antena, permite proponer la ecuación de síntesis (Ec.1) sujeto a $0.17 \lambda \leq L \leq 0.26 \lambda$.

$$
W \lambda^{-1} \approx 1.46\left(L \lambda^{-1}\right)^{2}-0.886\left(L \lambda^{-1}\right)+0.142
$$

En la Figura 4 se detalla la curva paramétrica de la directividad máxima en $\mathrm{dBi}$ de la antena meandro $\mathrm{N}=2$ versus la parte imaginaria de la impedancia de entrada de la antena. En esta gráfica se detalla el comportamiento de la radiación máxima de la antena considerando la modificación de los parámetros geométricos escogidos. Un análisis detallado de esta gráfica en la primera resonancia serie muestra que la directividad máxima de esta antena está en el rango de $1.7 \mathrm{dBi} \pm 0.2 \mathrm{~dB}$ y que su patrón de radiación es omnidireccional. Un análisis de esta curva considerando el ángulo de máxima radiación de la antena, y que no fue 


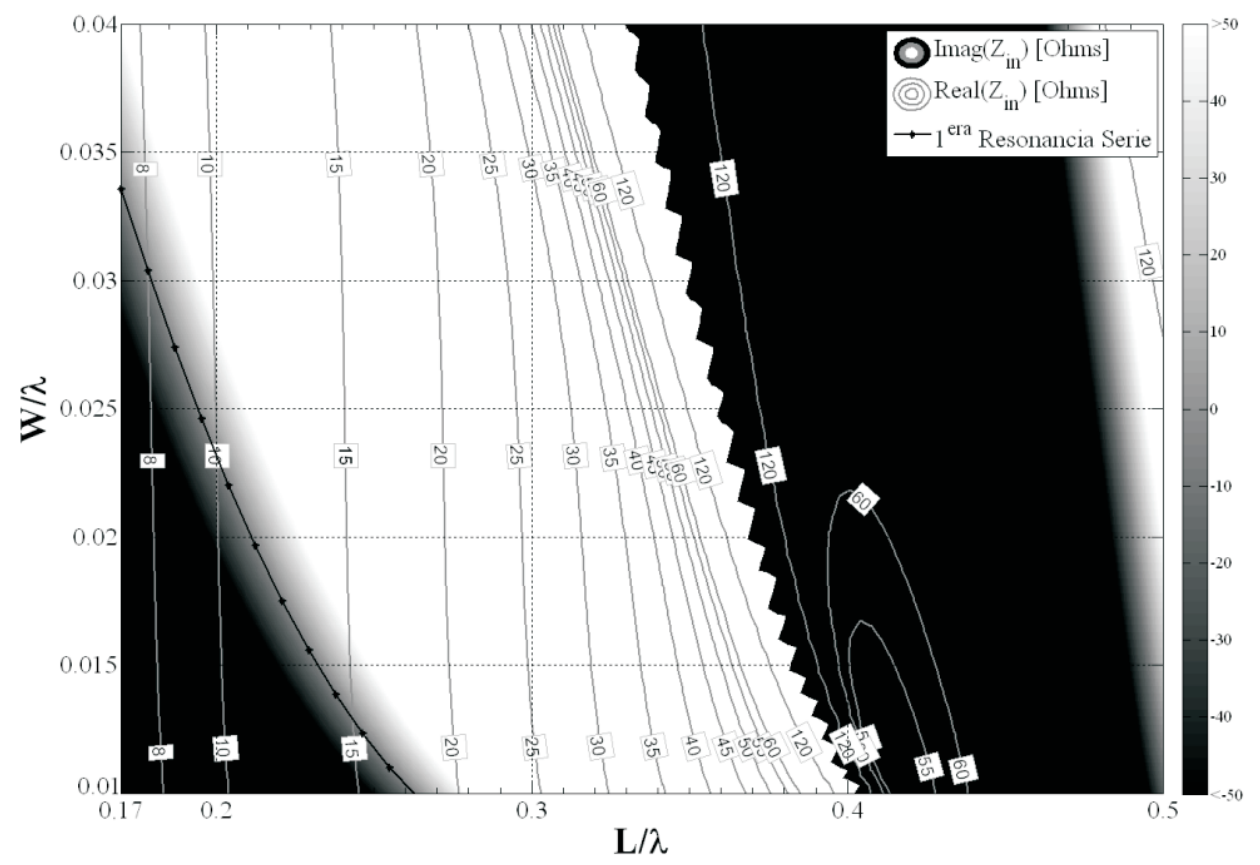

Figura 3. Gráfica paramétrica de la impedancia de entrada del dipolo meandro $N=2$

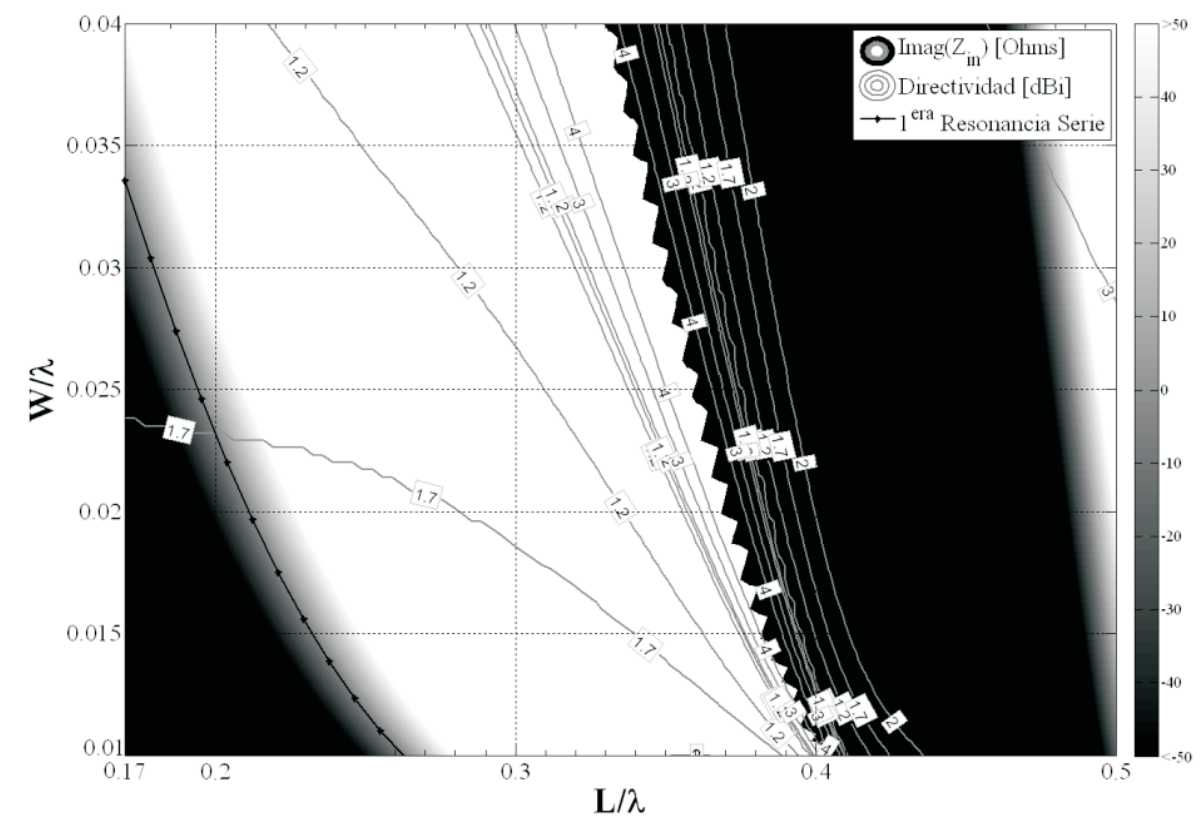

Figura 4. Gráfica paramétrica de la directividad máxima del dipolo meandro $N=2$ 
presentado en este documento por razones de espacio, muestra que en los alrededores de la primera resonancia paralela además de incrementarse la directividad máxima a más de 4 $\mathrm{dBi}$ y de tener una impedancia mucho mayor a 120 Ohmios, la dirección de máxima radiación se alterada, haciendo que la antena pase de ser omnidireccional a ser direccional.

\subsubsection{Curvas paramétricas del dipolo meandro $\operatorname{con} \mathrm{N}=4$}

En la Figura 5 se ilustra la curva paramétrica de la impedancia de entrada de la antena meandro $\mathrm{N}=4$, donde se detalla la primera resonancia serie y la primera resonancia paralela. La longitud total del dipolo meandro $\mathrm{N}=4$, como se deduce de la Figura 1 , es $2 L+2 \Delta$, y por ende, la capacidad de reducción de la longitud de esta antena graficada con respecto a un dipolo resonante $\lambda / 2$ esta en el rango de $34 \%$ y el $58 \%$. La ventaja del meandro $\mathrm{N}=4$ es que la impedancia de entrada está en el rango de $Z_{i n} \in[11 \Omega, 30 \Omega]$ y por ende, los dobleces adicionales incrementan la impedancia de entrada en esa resonancia, manteniendo la misma capacidad de reducción de tamaño que su equivalente $\mathrm{N}=2$. Un análisis gráfico de la relación entre W y L en la primera resonancia serie de esta antena, permite proponer la ecuación de síntesis (Ec.2) sujeto a $0.07 \lambda \leq L \leq 0.13 \lambda$.

$$
W \lambda^{-1} \approx 3.152\left(L \lambda^{-1}\right)^{2}-1.085\left(L \lambda^{-1}\right)+0.098
$$

En la Figura 6 se detalla la curva paramétrica de la directividad máxima en $\mathrm{dBi}$ de la antena meandro $\mathrm{N}=4$ versus la parte imaginaria de la impedancia de entrada de la antena. Un análisis detallado de esta gráfica en la primera resonancia serie muestra que el patrón de radiación es omnidireccional y la directividad máxima está en el rango de $1.85 \mathrm{dBi} \pm 0.05 \mathrm{~dB}$

\subsubsection{Curvas paramétricas del dipolo meandro con $\mathrm{N}=6$}

En la Figura 7 se ilustra la curva paramétrica de la impedancia de entrada de la antena meandro $\mathrm{N}=6$. En el rango de análisis solo se observa la primera resonancia serie y la primera resonancia paralela. La longitud total del dipolo meandro $\mathrm{N}=6$, como se deduce de la Figura 1, es $3 \mathrm{~L}+4 \Delta$, y por ende, la capacidad de reducción de la longitud de esta antena con respecto a un dipolo resonante $\lambda / 2$ esta en el rango de $34 \%$ y el $62 \%$. Como se detalla en la curva de la impedancia de entrada, el meandro con $\mathrm{N}=8$ continua incrementando el valor de la impedancia de entrada en resonancia, la cual está ahora en el rango de $Z_{\text {in }} \in[20 \Omega, 38 \Omega]$

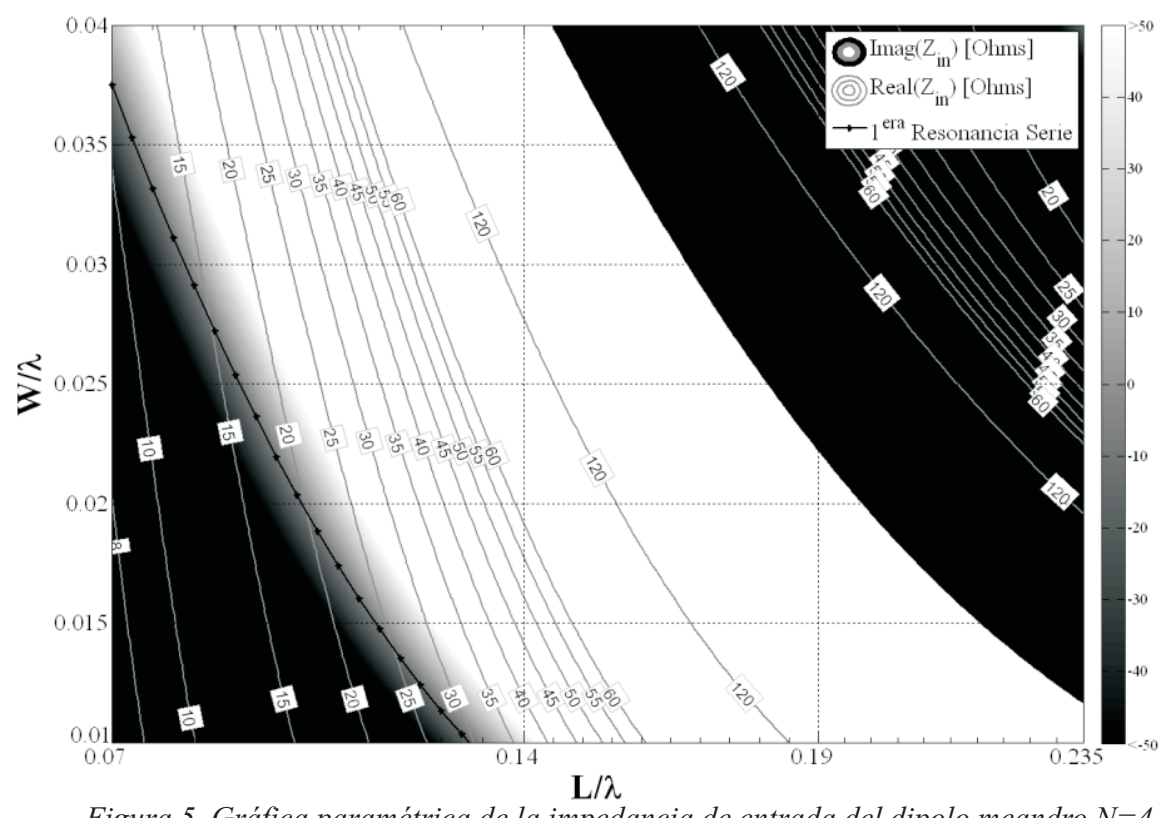

Figura 5. Gráfica paramétrica de la impedancia de entrada del dipolo meandro $N=4$ 


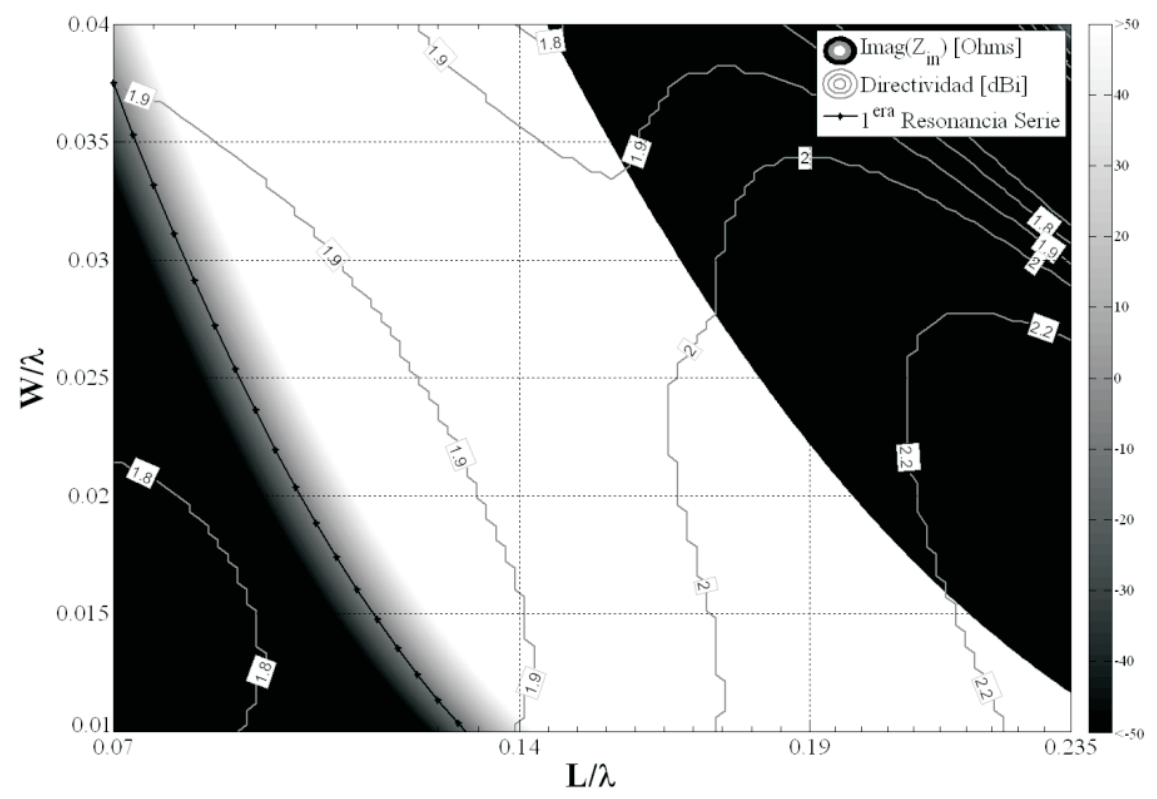

Figura 6. Gráfica paramétrica de la directividad máxima del dipolo meandro $N=4$

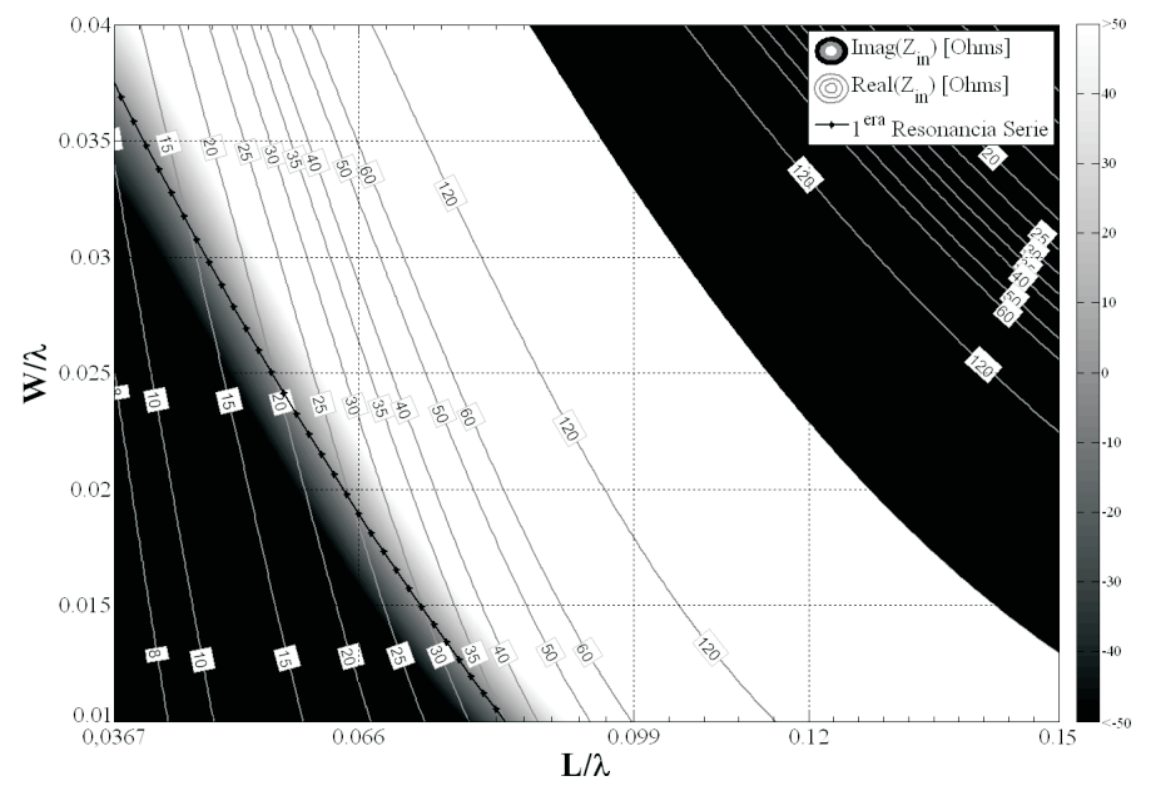

Figura 7. Gráfica paramétrica de la impedancia de entrada del dipolo meandro $N=6$ 
Un análisis gráfico de la relación entre $\mathrm{W}$ y L en la primera resonancia serie de esta antena, permite proponer la ecuación de síntesis (Ec.3) sujeto a $0.036 \lambda \leq L \leq 0.083 \lambda$

$$
W \lambda^{-1} \approx 2.634\left(L \lambda^{-1}\right)^{2}-0.902\left(L \lambda^{-1}\right)+0.067
$$

En la Figura 8 se detalla la curva paramétrica de la directividad máxima en $\mathrm{dBi}$ de la antena meandro $\mathrm{N}=6$ versus la parte imaginaria de la impedancia de entrada de la antena. Un análisis detallado de esta gráfica en la primera resonancia serie muestra que la directividad máxima de esta antena está en el rango de $2.05 d B i \pm 0.15 d B$ y que su patrón de radiación es omnidireccional.

\subsubsection{Curvas paramétricas del dipolo meandro $\operatorname{con} \mathrm{N}=8$}

Finalmente, la última configuración analizada fue la antena dipolo meandro con $\mathrm{N}=8$. En la Figura 9 se detalla la impedancia de entrada de este dipolo, en donde se presenta su primera resonancia serie y su primera resonancia paralela. Al igual que sus predecesores, a partir de la Figura 1 se deduce que la longitud total de este dipolo es de $4 L+6 \Delta$ y por ende, la capacidad de reducción de la longitud de esta antena con respecto a un dipolo resonante $\lambda / 2$ esta en el rango de $38 \%$ y el $57 \%$. Como se detalla en la curva de la impedancia de entrada, el meandro con $\mathrm{N}=8$ continua incrementando el valor de la impedancia de entrada en resonancia, la cual está ahora en el rango de $Z_{\text {in }} \in[20 \Omega, 38 \Omega]$

Un análisis gráfico de la relación entre $\mathrm{W}$ y L en la primera resonancia serie de esta antena, permite proponer la ecuación de síntesis (Ec.4) sujeto a $0.036 \lambda \leq L \leq 0.06 \lambda$

$$
W \lambda^{-1} \approx 2.869\left(L \lambda^{-1}\right)^{2}-0.899\left(L \lambda^{-1}\right)+0.053
$$

En la Figura 10 se detalla la curva paramétrica de la directividad máxima en $\mathrm{dBi}$ de esta antena meandro versus la parte imaginaria de la impedancia de entrada de la antena. Un análisis detallado de esta gráfica en la primera resonancia serie muestra que la directividad máxima de esta antena está en el rango de $2.05 \mathrm{dBi} \pm 0.1 \mathrm{~dB}$ y que su patrón de radiación es omnidireccional.

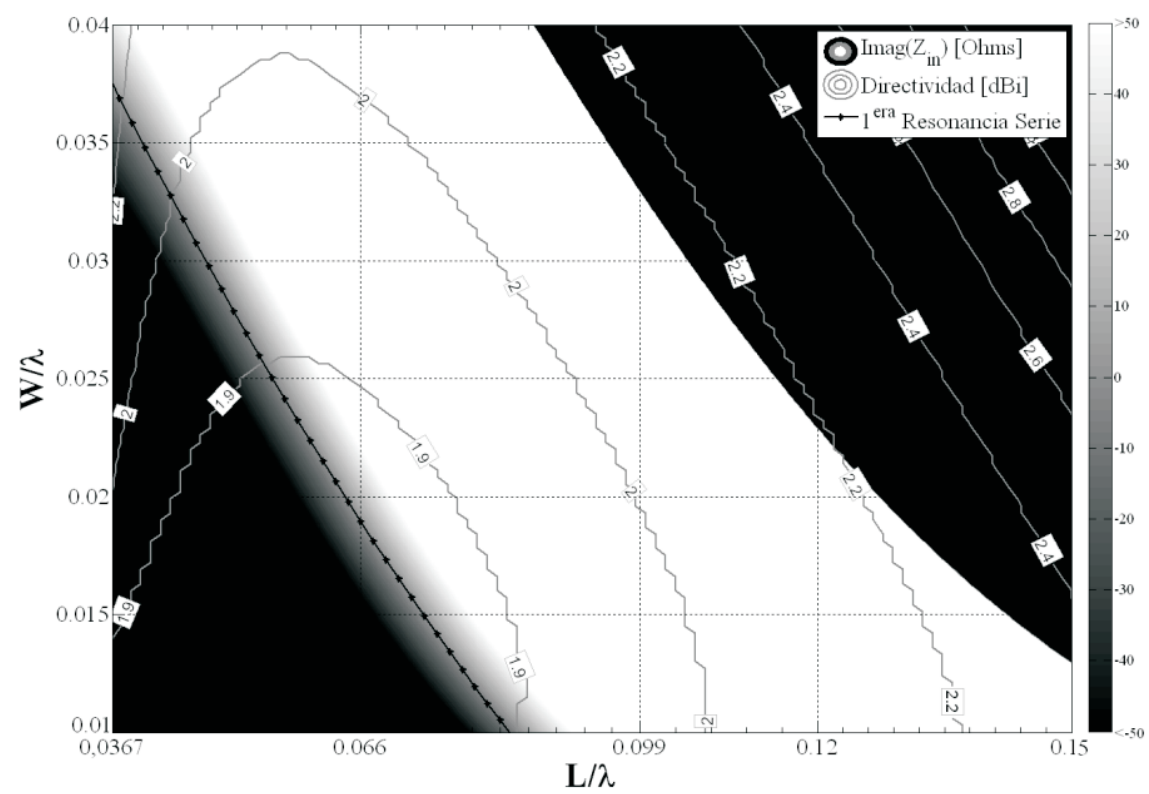

Figura 8 . Gráfica paramétrica de la directividad máxima del dipolo meandro $N=6$ 


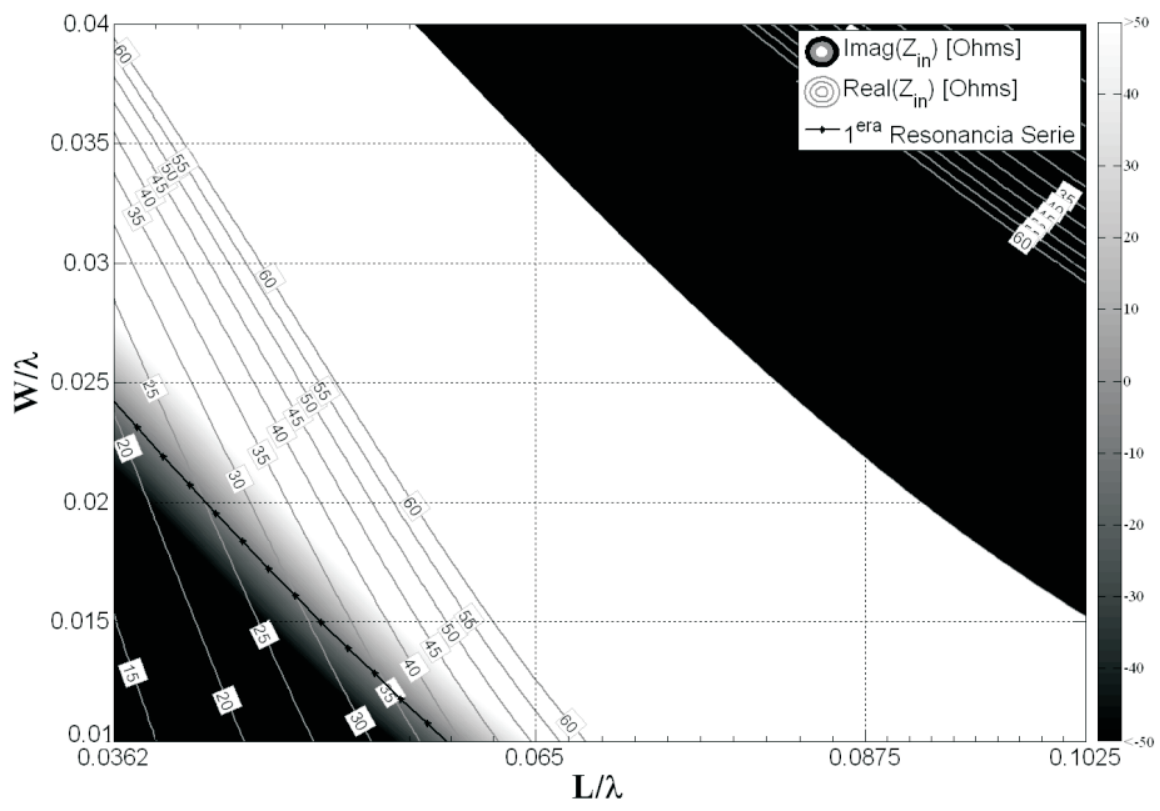

Figura 9. Gráfica paramétrica de la impedancia de entrada del dipolo meandro $N=8$

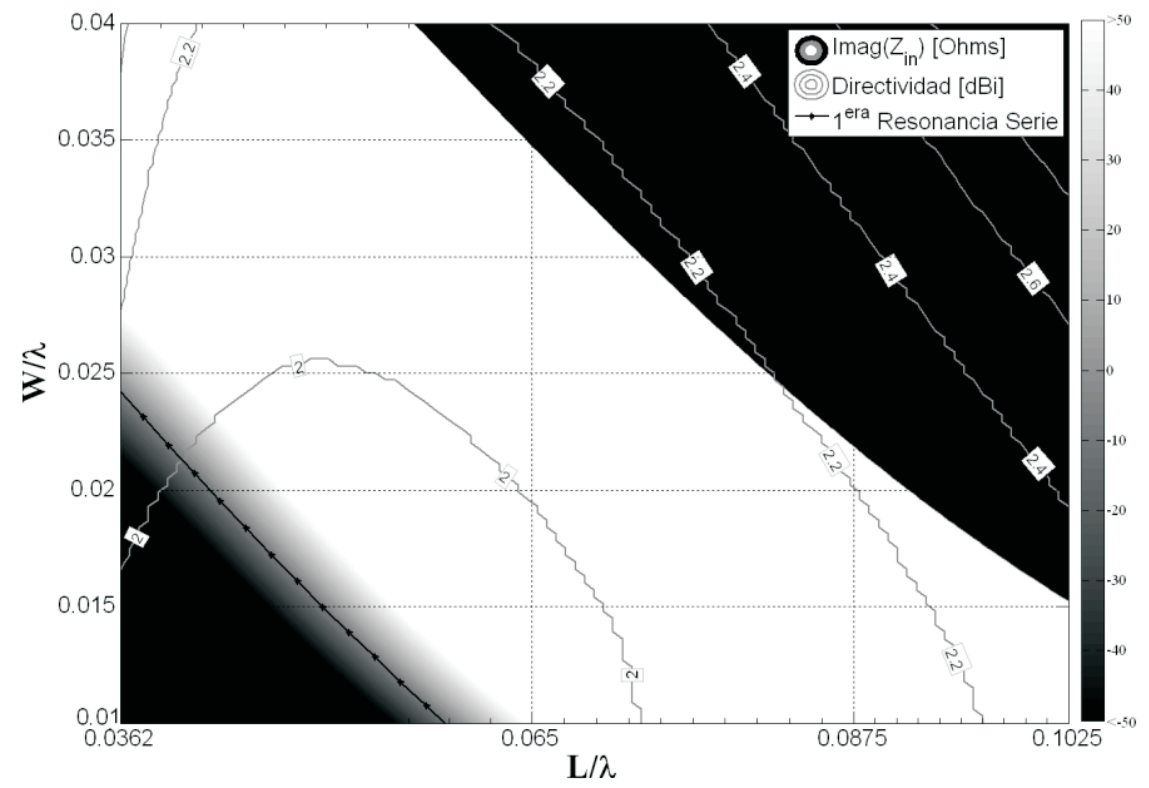

Figura 10. Gráfica paramétrica de la impedancia de entrada del dipolo meandro $N=8$ 
Como se deduce de las curvas de diseño presentadas, la impedancia de entrada de la antena meandro en la primera resonancia se encuentra en intervalos aceptables para la construcciones de redes de acople y para mantener una eficiencia de radiación alta. En todos los casos analizados, la antena operando en la primera resonancia serie tiene un patrón de radiación omnidireccional y presenta una directividad que en general se puede considerar en el intervalo $1.7 \mathrm{dBi} \leq D_{\max } \leq 2.2 \mathrm{dBi}$.

$\mathrm{El}$ incrementar el número de meandros $\mathrm{N}$ en cada brazo del dipolo no afecta considerablemente el porcentaje de reducción del tamaño de la antena y si mejora sustancialmente las posibilidades de la fabricación de las redes de acople, ya que la impedancia de entrada en esa resonancia se incrementa.

\subsection{Prototipos experimentales}

Con el fin de evaluar las curvas paramétricas presentadas y comprobar la hipótesis que la impedancia de entrada de la antena en la primera resonancia serie se incrementa entre más meandros se coloquen a una longitud total equivalente, se diseñaron y construyeron cuatro prototipos de las antenas meandro a una frecuencia de operación de $150 \mathrm{MHz}$. Los puntos de operación fueron escogidos con el fin de operar en la primera resonancia serie y lograr una reducción del tamaño al 50\% comparado con un dipolo resonante Figura 11. Las antenas fueron construidas sin equipos especializados y con alambre de aluminio de diámetro $4.76 \mathrm{~mm}$ y sus largos variaron entre 52 centímetros y 58 centímetros. En el punto de alimentación se utilizó un Balun ferrita 1:1 de montaje superficial en configuración de corriente soldado en un substrato FR-4 con conectores BNC.

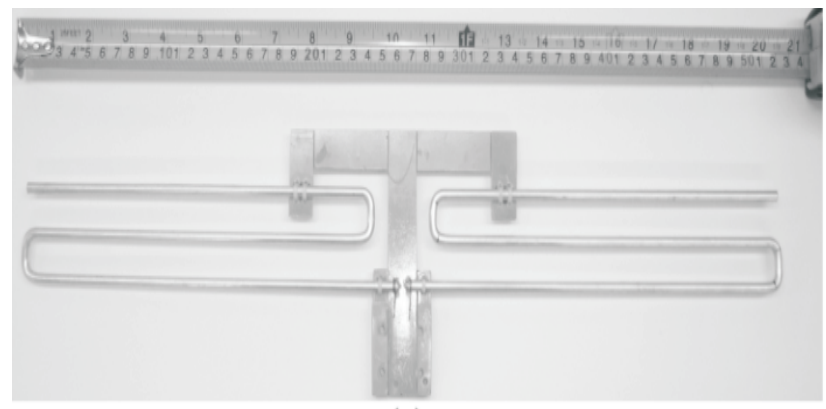

(a)

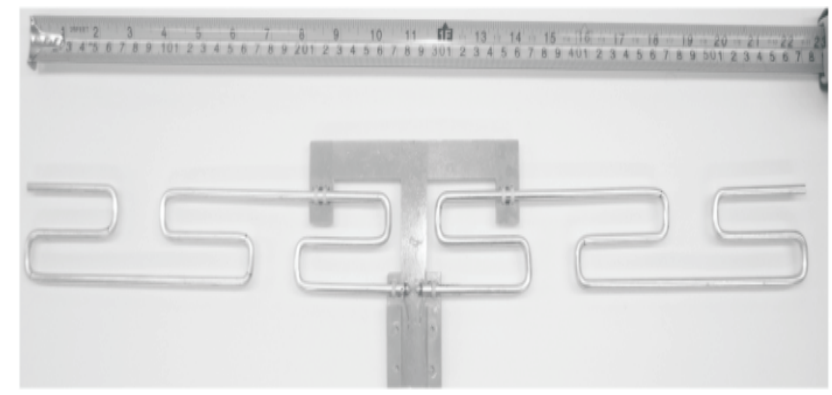

(c)

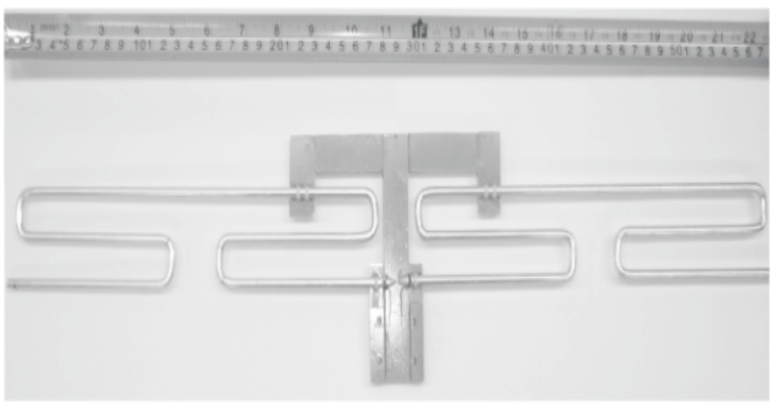

(b)

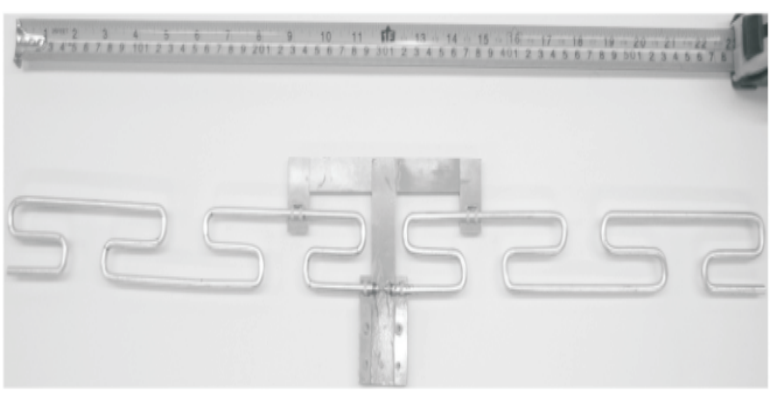

(d)

Figura 11. Prototipos de las antenas meandro construidas. (a) Antena meandro $N=2$. (b) Antena meandro $N=4$. (c) Antena meandro $N=6$. (d) Antena meandro $N=8$. 


\subsection{Impedancia de entrada}

En la Figura 12 se detalla la impedancia de entrada de la antena, tanto en la parte real como en la parte imaginaria. Como se detalla en las curvas de la parte real de la impedancia de entrada en la frecuencia de la resonancia serie, se comprueba que entre más meandros contenga el dipolo mayor será la impedancia de entrada manteniendo su capacidad de miniaturización. Aunque las antenas prototipos construidas fueron diseñadas para operar en $150 \mathrm{MHz}$, las pruebas de laboratorio mostraron que la primera resonancia serie se presentó en $141 \mathrm{MHz} \pm 1 \mathrm{MHz}$.

La variación porcentual en la frecuencia de resonancia serie es del $6 \%$ y consideramos que fue debida a las imperfecciones de los modelos construidos, específicamente a los dobleces de los diferentes meandros.

Los valores experimentales de la impedancia de entrada en la primera resonancia serie fueron de $11.9 \Omega, 17.2 \Omega, 18.5 \Omega$ y $21 \Omega \quad$ para las antenas meandro $\mathrm{N}=2, \mathrm{~N}=4, \mathrm{~N}=6$ y $\mathrm{N}=8$, respectivamente. Los valores teóricos encontrados a partir de las curvas presentadas en este documento, son de $15.7 \Omega, 21.7 \Omega, 24.1 \Omega$ y $23.5 \Omega$ para las antenas meandro $\mathrm{N}=2, \mathrm{~N}=4, \mathrm{~N}=6$ y $\mathrm{N}=8$, respectivamente. Aunque el error relativo en el peor de los casos es del $32 \%$, realmente el error numérico no supera el valor de $5.6 \Omega$ lo cual es aceptable considerando el rango de valores de la impedancia que se espera tener en la antena en los puntos de resonancia escogidos. Al igual que el corrimiento de la frecuencia de resonancia, nosotros consideramos que el error presentado fue debido a las imperfecciones de los modelos construidos, específicamente a los dobleces de los diferentes meandros y a la imposibilidad experimental de detallar el comportamiento del Balun utilizado. El error entre los valores de la parte imaginaria de la impedancia de entrada predicho y medido fue despreciable.

\subsection{Patrón de radiación}

En la Figura 13 se detalla el patrón de radiación E de las diferentes configuraciones meandro, en donde por aspectos comparativos se presenta la curva teórica encontrada con el simulador y los diferentes patrones de radiación en un barrido en frecuencia entre el $95 \%$ y el $103 \%$ de la primera frecuencia de resonancia serie. Como se observa en las gráficas, el patrón de radiación sigue el valor esperado por el simulador excepto en los nulos, localizados en 90 grados y 270 grados. Esta situación es debido a que el muestreo angular experimental en el laboratorio utilizado tiene una resolución de \pm 5 grados, haciendo que los nulos tengan un error de predicción importante dado su gran selectividad angular.

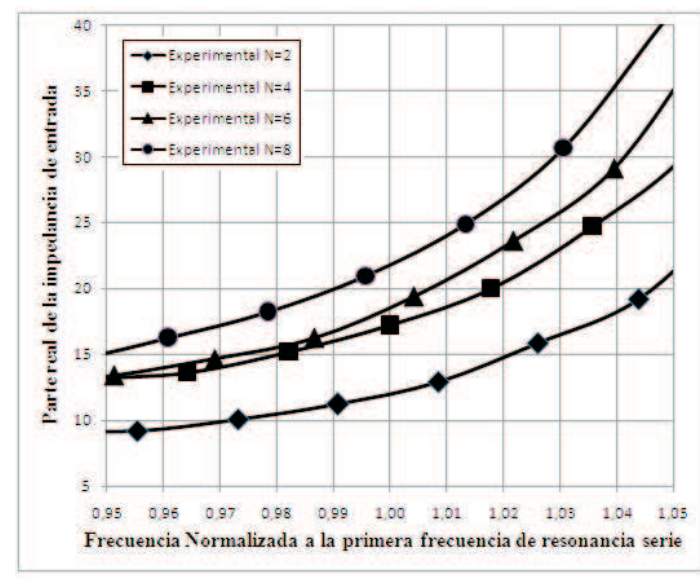

(a)

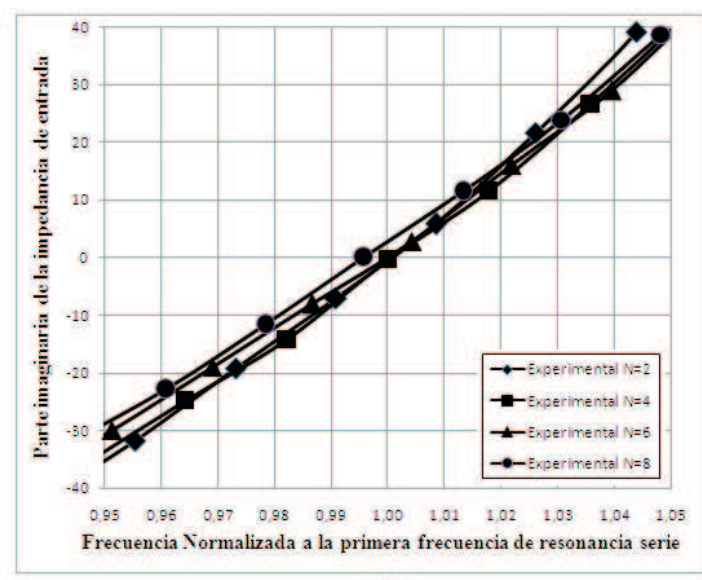

(b)

Figura 12 . Impedancia de entrada del dipolo meandro con respecto a la frecuencia. (a) Parte real de la impedancia de entrada. (b) Parte imaginaria de la impedancia de entrada. 


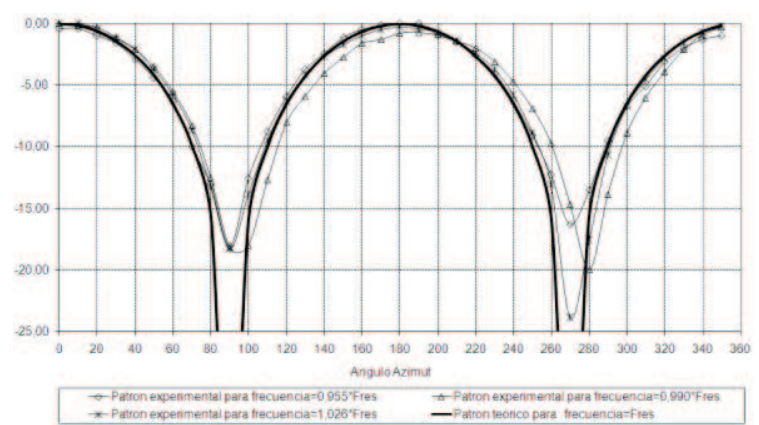

(a)

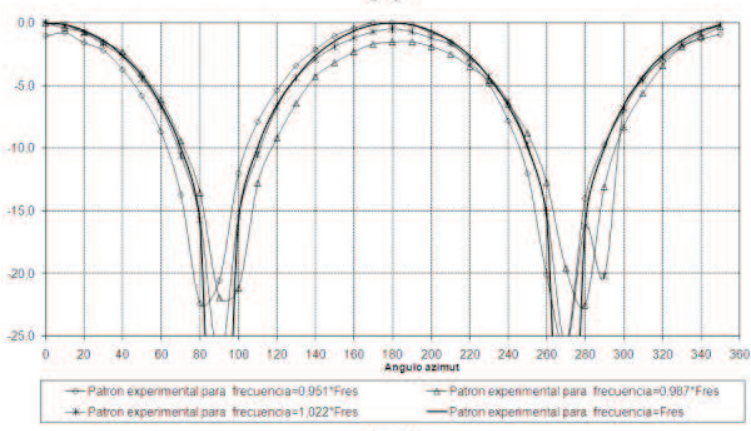

(c)

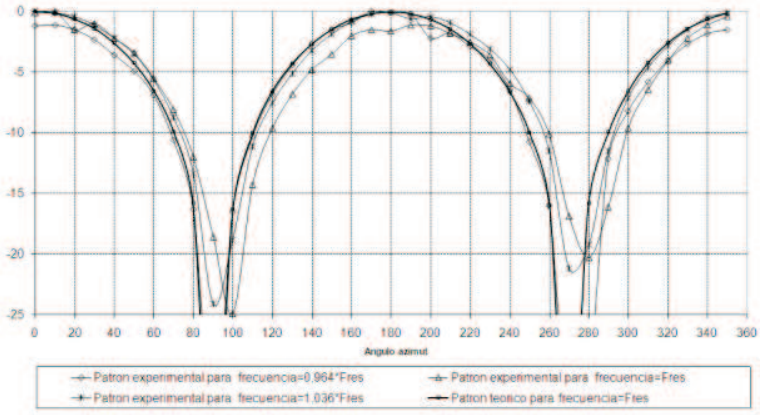

(b)

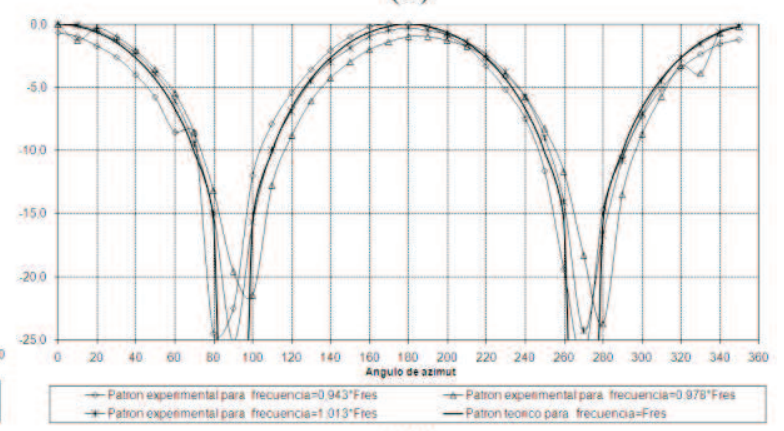

(d)

Figura 13. Patrón de radiación de cada antena meandro en el plano E. (a) Antena meandro $N=2$. (b) Antena meandro $N=4$. (c) Antena meandro $N=6$. (d) Antena meandro $N=8$.

El patrón de radiación vertical también fue analizado y debido a su comportamiento constante en el barrido angular no fue presentado en este documento. En estas gráficas se presentó una variación de $2 \mathrm{~dB}$ con respecto al valor teórico, debido a la influencia del mástil metálico en el cual se realizaron las mediciones experimentales.

\subsection{Ganancia de la antena}

La ganancia máxima encontrada en el patrón E en las diferentes antenas meandros estuvo en los rangos de $0.46 d B i \leq G_{E} \leq 1 d B i$.

Esto quiere decir que el error general entre la medida obtenida y la medida predicha a través de las curvas paramétricas tiene un error máximo de $1.2 \mathrm{~dB}$. Aún lo anterior, la ganancia obtenida está dentro de un rango aceptable para su operación.

\section{Conclusiones}

En la presente investigación se han desarrollado curvas de diseño para la antena de alambre en configuración meandro, a partir de la caracterización electromagnética de la estructura en NEC. Diversas configuraciones de la antena fueron comprobadas experimentalmente en un puntos de operación que garantizan una reducción del tamaño de la antena a un cincuenta por ciento $(50 \%)$ comparado con una antena resonante tradicional.

Los resultados muestran que los intervalos de miniaturización para las diferentes configuraciones es aproximadamente el mismo para las diferentes configuraciones del meandro, con la ventaja que un incremento en la cantidad de curvas en cada brazo del dipolo, incrementa la impedancia de la entrada de la antena en la primera resonancia serie, y por ende, se incrementa la 
factibilidad de construir redes de acople más sencillas y de incrementar la eficiencia de la antena.

La reducción encontrada con las antenas analizadas está en el rango entre el $34 \%$ y $62 \%$ del largo de cada brazo de la antena, el cual es satisfactorio y comparable con la reducción de otro tipo de antenas más sofisticadas, como las antenas fractales en configuración Koch, con la ventaja de una simplificación importante en su geometría y por ende en su construcción. Aún la antena meandro más simple, cuya configuración es $\mathrm{N}=2$, se obtiene en la primera resonancia serie una reducción considerable en la longitud de la antena (34\%) con un muy razonable valor de impedancia de entrada en resonancia $(7 \Omega)$.

\section{Agradecimientos}

Deseamos agradecer a la Pontificia Universidad Javeriana y en especial al Departamento y Laboratorio de Electrónica por sus aportes en la elaboración y realización de las pruebas experimentales requeridas para finalizar este proyecto. También deseamos agradecer al Director de la maestría en electrónica, por la financiación del estudiante de maestría relacionado en este proyecto de investigación. Finalmente, deseamos agradecer al estudiante de pregrado Jose Joaquín Casas por sus aportes en la construcción de los prototipos de las antenas y apoyo en la toma de datos experimentales.

\section{Referencias bibliográficas}

Altshuler, E.E. \& Linden, D.S.(1997), Design of a loaded monopole having hemispherical coverage using a genetic algorithm. IEEE Transactions on Antennas and Propagation, 45 (1),1-4.

Altshuler, E.E. (2002). Electrically small selfresonant wire antennas optimized using a genetic algorithm. IEEE Transactions on Antennas and Propagation. 50 (3), 297-300.
Baliarda, C.P. Romeu, J. \& Cardama, A. (2000). The Koch monopole: a small fractal antenna. IEEE Transactions on Antennas and Propagation. 48(11), 1773-1781.

Balanis, C.A. (1997). Antenna Theory: Analysis and Design. Second Edition, John Wiley and Sons, New York.

Best, S.R. (2003). On the performance properties of the Koch fractal and other bent wire monopoles. IEEE Transactions on Antennas and Propagation. $51(6), 1292-1300$.

Boag, A. Michielssen, E. \& Mittra, R. (1996). Design of electrically loaded wire antennas using genetic algorithms. IEEE Transactions on Antennas and Propagation, 44(5), 687-695.

Burke, G. J. \& Poggio, A. J. (1981). Numerical Electromagnetics Code (NEC)_Method of Moments. Lawrence Livermore Laboratory, Livermore, CA, UCID18834.

Erentok, A., \& Ziolkowski, R.W. (2008). Metamaterial-Inspired Efficient Electrically Small Antennas. IEEE Transactions on Antennas and Propagation, 56(3), 691-707.

Galehdar, A., Thiel, D.V.,\& O'Keefe, S.G. (2009). Tapered Meander Line Antenna for Maximum Efficiency and Minimal Environmental Impact. IEEE Antennas and Wireless Propagation Letters, $8,244-247$.

Gonzalez-Arbesu, J.M. Blanch, S. \& Romeu, J. (2003). Are space-filling curves efficient small antennas?, IEEE Antennas and Wireless Propagation Letters, 2, 147-150.

Hosung Choo, Rogers, R.L.,\& Hao Ling. Design of electrically small wire antennas using a pareto genetic algorithm. IEEE Transactions on Antennas and Propagation, 53(3), 1038-1046.

Jinhui Zhu. Hoorfar, A. \& Engheta, N. (2004). Peano antennas. IEEE Antennas and Wireless Propagation Letters. 3, 71-74. 
Petko, J.S. \& Werner, D.H.(2004). Miniature reconfigurable three-dimensional fractal tree antennas. IEEE Transactions on Antennas and Propagation. 52(8), 1945-1956.

Rashed-Mohassel, J. Mehdipour, A. \& Aliakbarian, H. (2009). New schemes of size reduction in space filling resonant dipole antennas. 3rd European Conference on Antennas and Propagation, EuCAP 2009, 2430-2432.

Rashed, J.,\& Tai, C.-T. (1991). A new class of resonant antennas. IEEE Transactions on Antennas and Propagation, 39 (9), 1428-1430.

Simpson, T.L. (2004). The disk loaded monopole antenna. IEEE Transactions on Antennas and Propagation, 52 (2), 542-550.

Warnagiris, T.J. \& Minardo, T.J. (1998). Performance of a meandered line as an electrically small transmitting antenna. IEEE Transactions on Antennas and Propagation. 46(12),1797-1801.
Werner, D.H. \& Ganguly, S. (2003). An overview of fractal antenna engineering research. IEEE Antennas and Propagation Magazine. 45(1), 3857.

Wen-Yan Yin. Yue Ping Zhang. Guo Xin Zheng. Mei Sun. (2009). Performance of Intra-Chip Wireless Interconnect Using On-Chip Antennas and UWB Radios. IEEE Transactions on Antennas and Propagation. 57(9), 2756-2762.

Yamada, Y. \& Michishita, N. (2005). Antenna efficiency improvement of a miniaturized meander line antenna by loading a high \&epsiv;r material. IEEE International Workshop on Antenna Technology: Small Antennas and Novel Metamaterials. IWAT 2005, 159-162. 